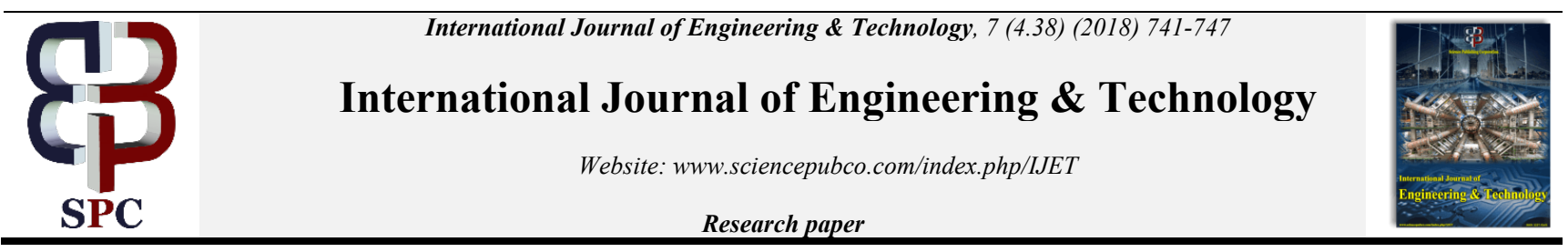

\title{
Modelling of Heat and Mass Transfer for Wire-Based Additive Manufacturing Using Electric Arc and Concentrated Sources of Energy
}

\author{
Dmitriy Trushnikov ${ }^{1}$, Anatoly Perminov ${ }^{1}$, Shengyong Pang ${ }^{2}$, K.P. Karunakaran ${ }^{3}$, Vladimir Belenkiy ${ }^{1}$, Gleb \\ Permyakov ${ }^{1}$, Maksim Kartashov ${ }^{1}$, Evgeniy Matveev ${ }^{1}$, Alena Dushina ${ }^{1}$, Yury Schitsyn ${ }^{1}$ \\ ${ }^{1}$ Perm National Research Polytechnic University, Perm, Russia \\ ${ }^{2}$ Huazhong University of Science and Technology, Wuhan, China \\ ${ }^{3}$ Indian Institute of Technology Bombay, Mumbai, India \\ *Corresponding author E-mail: trushnikov.dm@mail.ru
}

\begin{abstract}
The paper presents a model developed by the authors and aimed to describe heat and mass transfer during wire-based additive manufacturing, when electron beam, plasma or arc are used as energy sources in case of non-consumable electrode welding. The model describes non-stationary and non-equilibrium conjugated processes of heat and mass transfer in free-surface liquid metal. The solution of differential equations of viscous fluid motion (Navier-Stokes), with convective terms and at laminar flow, has become the model base. Melting and crystallization of the metal is recognized by heat release in a two-phase region. The material density variation during phase transitions of the first and the second order can be described by introducing a certain dependence on temperature. The model is able to consider the use of preliminary and additional induction heating by changing the initial temperature and establishing an additional distributed bulk heat source. Variables for the simulation of heat and mass transfer during additive formation are the intensity and type of the heat source, the plate initial temperature, the power density distribution, the intensity of the additional bulk heating, the dependence of material thermal and physical characteristics on temperature, the characteristics of the phase transitions, the motion velocity of the heat source, the rate of wire feeding.
\end{abstract}

Keywords: additive manufacturing, 3D wire-based deposition, arc and concentrated heat sources, modelling of heat and mass transfer, numerical implementation.

\section{Introduction}

Nowadays, the development of mathematical modelling methods leads to a wide use of computer technologies at the stage of machinery preproduction engineering. Introduction of a digital production concept based on the modelling of manufacturing processes using hybrid technologies is one of the most important issues of the industrial enterprises development. In the recent decade, the industrial enterprises have been largely using additive technologies (e.g. fusion welding) that utilise such sources of energy as electric arc, plasma jet, laser or electron beams, and filler wire as source material [1-4]. The use of these technologies is most suitable for production of large parts. Modelling the processes of the hybrid manufacturing solutions based on product formation in layers by melting the wire filler with electric arc or concentrated heat sources (plasma arc, electron or laser beam) makes it possible to pre-select technological parameters of the additive processes and significantly reduce the stage of the manufacturing process development.

The control of 3D wire-based deposition is a complex task. Cross dimensions and forms of weld beds, metallurgical processes and material structures to be formed will depend on many factors, most of which can hardly be controlled. All these characteristics of a deposited layer are directly connected to the parameters characterizing heat and mass transfer, such as vector field of velocities and scalar fields of temperature, pressure and density. Apparently, only a small part of the parameters can be determined experimentally. It defines the need in creating a computer model of the process. Heat and mass transfer mathematical model for wire-based additive process should generally describe phase transitions, heterogeneous thermal and physical properties of the materials, and take into account Marangoni effect at the interface between two phases.

Additive manufacturing has much in common with welding and allied processes. A moving source of heat creates a weld bed, which moves together with the heat source. From the technological point of view, additive technologies are much closer to multilayer deposition that is also characterized by the source material's (filler wire) interaction with the heat source, the gradual growth of layers. It also requires considering a number of specific aspects to better understand the additive technologies. As a rule, it is difficult to determine distribution of the thermal fields in a processed piece using experimental methods. The use of thermocouples and imagers makes it possible to read only surface temperatures, while mathematical modelling could determine distribution of the piece bulk temperatures.

The majority of the models available for heat and mass transfer related to additive technologies are developed using a number of simplifications. In particular, in order to simplify calculations in [5. 6], the authors employed a two-dimensional setting, while in 
$[7,8]$ the convective heat transfer in the melt has not been considered despite the fact that the convective flows move the liquid metal, and the nature of circulation has a significant effect on temperature distribution in the liquid metal, heating and cooling rate, crystallization process, material microstructure and mechanical properties [9]. At the same time, the precise calculation will require a solution of a coupled equation of thermal conductivity and melt flow. We assumed that density and other thermal and physical characteristics of the material in solid and liquid states are constant, as this assumption helps to save computational time. Surfaces of deposited layers are often approximated to simple figures and considered to be plane. This assumption does not largely influence thermal fields and cooling rate. Thermal effects associated with evaporation of alloying elements are also ignored, as the effect is usually minor compared to the incoming energy from the heat source. One of most frequently used methods aimed at measuring temperature is to put thermocouples in the vicinity of the heat source area [10-12] Thermocouples are not good temperature field registration. Infrared observation [13 -17] is also used to measure the distribution of transitional temperature processes in bulk. So an alternative method involves the estimation of temperature fields and cooling rates using mathematical models after their validation with the help of the experimental data containing the relations of temperature and time at several monitoring points

In order to model heat and mass transfer in different additive technologies, researchers use the numerical and analytical method, which helps them to calculate fields of velocities, temperatures, free surface motion, phase boundaries etc. The numerical modelling of the motion of a two-layer system is based on the Level-Set method, which major principles are given in [18-20]. An example of successful applications of the Level-Set method used to determine the position of the liquid-gas boundary phase can be found in [21-23].

The following conservation equations for mass, momentum and energy are usually used [24-28]. The convective motion of the melt in a weld pool is calculated using the equation for the surface-tension gradient on the melt surface as a result of the temperature gradient (Marangoni convection) [27, 28].

The variables for the simulation at the wire-based deposition are as follows:

- heat source intensity;

- type of heat source (electric arc, plasma arc, laser emission, etc.);

- density distribution of the heat source energy flow;

- initial temperature of the sample;

- the material thermal and physical characteristics on temperature;

- phase transformation parameters;

- velocity of a heat source motion;

- wire diameter and feeding.

The following assumptions have been made as part of the mathematical models described below:

- the intensity of the heat source, speed of movement of the heat source and wire feed speed are stable;

-the temperature of ambient is invariable;

- the heat source is Gaussian. An explicit form of the intensity change depending on the depth is to be determined;

- $\quad$ the materials of the plate and wire are the same;

- liquid metal is assumed to be an incompressible liquid

- thermophysical variables do not determined by on temperature.

- the effective heat capacity is used to take into account thermal effects during melting and solidification;
- the additional force in the equation of the motion is used to take into account the processes at a two-phase region on the melting pool boundary;

- an influence of arc electric and magnetic fields to melt transportation is not take into consideration.

\section{Model Description}

The deposition by concentrated sources of energy employs nonsymmetric wire feed as in the scheme, presented in Fig. 1.

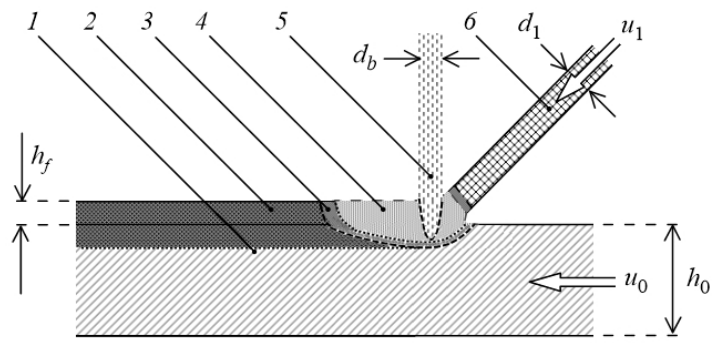

Fig.1: Principal scheme of the deposition by concentrated sources with a side wire feed: 1) a pre-deposit and substrate; 2) the deposited weld bed; 3 ) a two-phase zone; 4) a liquid phase; 5) electron, laser beam or plasma arc; 6) wire

The filler wire is supplied at a certain angle into the heat source effect zone. Electron beam, laser beam, plasma arc or electric arc with a non-consumable electrode can be used as a heat source. Due to the impact of the source of thermal energy, the metal melts and evaporates. The surface of the liquid bath may be distorted the shape by the of plasma arc pressure, vapour pressure and falling drops. The technological parameters of this process effect to the liquid metal transfer from the wire.

There are several metal transfer regimes possible [29]:

- spray metal transfer, which requires a low intensity heat source, and is considered to be a relatively low-temperature mode. In such a case, the sizes and the form of the weld bed cross section, as well as the structure and physical and mechanical properties of the deposited metal will vary insignificantly during the process. A further reduction in heat input causes an incomplete melting of the wire, which generates defects and, therefore, the regime is invalid;

- the coarse-droplet transfer, which requires an increase of the heat source intensity up to the critical value. At this metal transfer mode, the form of the weld differ along the product length. Strong waviness and spatter are present. Crystallization is unstable. This transfer mode can to be used for MIG welding technology, but its use in most cases leads to a decrease in quality during the additive formation processes;

- a fine-droplet transfer requires even higher intensity of the heat source. The metal being constantly fed is exposed to the heat that is sufficient to induce the intensive boiling of this metal. The features of the mode are high spatter and uneven surface of the deposited rollers.

The weld bed formation is based on solving both the heat and the hydrodynamic problems in a three dimensional setting. Fig. 2a presents the weld bed formation by wire filler melted and a heat source. Fig. $2 b$ shows a longitudinal section of a computational region in a plane of symmetry. The symmetry allows including only half of the whole object into the computational region when describing the formation of a straight-line weld bed on a substrate. 


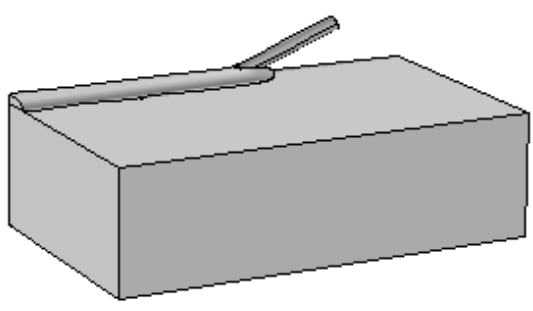

a)

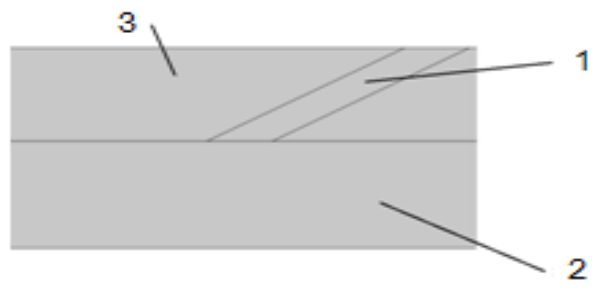

b)

Fig.2: The scheme of wire-based weld bed formation: (a) longitudinal section of the computational region in a plane of symmetry; (b) 1 is the fed wire material; 2 is metal; 3 is gas environment

The shock capturing method is proposed to solve this challenge. The method implies solving the motion and temperature distribution equations not only for the metal, but also for the gas environment located above the metal and deposition zone. To describe the position of the metal-gas interface, the Level-Set method was implemented. This method makes it possible to trace the free surface of the weld bed. Three dimensional distributions of weld bed temperature and velocity with the free surface are taken as the outcoming data, while the calculated form and size of beds are in agreement with the experimental data. However, the method entails high requirements for computational resources [21, $30-32]$. To calculate the position of the interface and the values of environment variables the level-set function $\phi$ was used, which is determined from the solution of a separate equation.

The phase transition surface during melting and crystallization is determined by the melting isotherm. The motion of the molten metal is described by the equations of motion with regard to thermal convection for incompressible liquid:

$\frac{\partial}{\partial t}(\rho \vec{u})+(\vec{u} \nabla)(\rho \vec{u})=-\nabla P+\operatorname{Div} \overline{\bar{\tau}}+\vec{F}$

$$
\operatorname{div} \vec{u}=0
$$

$$
\tau_{i j}=\mu\left(\frac{\partial u_{i}}{\partial x_{j}}+\frac{\partial u_{j}}{\partial x_{i}}\right)
$$

where $\rho$ is the density, $\vec{u}$ is the melt flow rate vector, $\mu$ is the dynamic viscosity, $P$ is the pressure.

The item $\vec{F}$ in equation (1) for the computational region 1 in Fig. $2 \mathrm{~b}$ will be as follows

$$
\vec{F}=\rho \vec{g} \beta\left(T-T_{r e f}\right)-C\left(\frac{1-f_{L}}{f_{L}^{3}+B}\right)\left(\vec{u}-\vec{u}_{1}\right)+\overrightarrow{\mathrm{F}}_{\mathrm{sv}}
$$

for regions 2 and 3 (Fig. 2b)

$$
\vec{F}=\rho \vec{g} \beta\left(T-T_{r e f}\right)-C\left(\frac{1-f_{L}}{f_{L}^{3}+B}\right)\left(\vec{u}-\vec{u}_{0}\right)+\overrightarrow{\mathrm{F}}_{\mathrm{sv}},
$$

where $\vec{u}_{0}$ is the substrate feeding rate, $\vec{u}_{1}$ are the wire feeding rates, $g$ is the gravitational acceleration, $\beta$ is the thermalexpansion coefficient, $T$ is the absolute temperature, $T_{r e f}$ is the reference temperature given as the temperature of the solidus $\left(T_{S}\right)$. The first part of equations (2) and (3) describes thermogravity convection phenomena, the second term describes the heat release in a two-phase region, where $B$ is implemented to eliminate dividing to $0, C$ is a parameter and can be taken equal to
$10^{4} \ldots 10^{6}, f_{L}$ is a parameter that determines the interface between the liquid and solid phases of the material:

$f_{L}=\left\{\begin{array}{lc}1 & T>T_{L} \\ \left(T-T_{S}\right) /\left(T_{L}-T_{S}\right) & T_{S} \leq T \leq T_{L} \\ 0 & T<T_{S}\end{array}\right.$

where $T_{L}$ and $T_{S}$ are liquidus temperature and solidus temperature, respectively.

The third term of equation (2) and (3) is the volume representation of forces acting on the interface.

The temperature distribution is determined from the solution of the differential heat equation:

$$
\frac{\partial T}{\partial t}+(\vec{u} \nabla) T=a \cdot\left(\frac{\partial^{2} T}{\partial x^{2}}+\frac{\partial^{2} T}{\partial y^{2}}+\frac{\partial^{2} T}{\partial z^{2}}\right)+\frac{Q}{c_{p} \rho}
$$

where $T$ is the absolute temperature, $a=\lambda / C_{\text {eff }} \cdot \rho$ is the thermal diffusivity coefficient, $u$ is the melt fluid velocity, $c_{p}$ is the heat capacity at a constant pressure, $\rho$ is the density.

When describing the density of the heat flow at the interface of the equation (5), the expression for $Q$ has the following form:

$$
Q=\left(q_{r} n_{z}-q_{h}\left(T-T_{0}\right)-\sigma_{c \sigma} \varepsilon_{u}\left(T^{4}-T_{0}^{4}\right)\right) \delta(\phi),
$$

where $T_{0}$ is the ambient temperature far from the deposition zone; $\sigma_{c \sigma}$ is the Stefan-Boltzmann constant, $\varepsilon_{u}$ is the metal emissivity factor, $\delta(\phi)$ and $n_{z}$ are the delta-function and projection of the normal to the metal-gas boundary along $\mathrm{Z}$ axis, establishing the heat distribution along the metal-gas interface. They will be determined later when describing the Level-Set method.

The density of the heat flow from the welding source in this setting is given in the relative coordinates:

$$
q_{r}=\eta_{0}\left(\frac{2 Q_{0}}{\pi r^{2}}\right) \exp \left(\frac{-2\left(\left(x-x_{0}\right)^{2}+\left(y-y_{0}\right)^{2}\right)}{r^{2}}\right)
$$

where $Q_{0}$ is the maximal heat intensity of the power source, $r$ is the radius of the source, $\eta_{0}$ is the efficiency of the heat source. The type of the heat source, its intensity modulation or introduction of high-frequency spatial vibrations, e.g. oscillations of the electron beam, were taken into account by setting a corresponding averaged spatial distribution of the energy flow density. Also in case of plasma arc, the spatial distribution of energy flow density is influenced by its polarity (direct or reverse). 
The quasi-equilibrium model [33] is taken as the crystallization (melting) model, within which the solid phase in the two-phase (transient) zone is distinguished by the linear law. This fact determines the type of function $f_{L}$ in equation (3). Heat of fusion or crystallization in equation (4) is determined by the introduction of the effective heat capacity, which under these conditions increases abruptly within the interval of the two-phase zone temperature:

$C_{\text {eff }}= \begin{cases}C_{0} & T<T_{S}, T>T_{L} \\ C_{0}+\frac{L_{m}}{T_{L}-T_{S}} & T_{S} \leq T \leq T_{L} .\end{cases}$

where $C_{0}$ is the heat capacity, in the general case, depending on temperature, $L_{m}$ is the specific heat of phase transition (specific heat of fusion). During the calculations it is possible to replace the piecewise constant function of the effective heat capacity (8) with the smooth function

$$
C_{e f f}=C_{0}+\frac{\exp \left[-\left(\left(T-T_{S L}\right) /\left(T_{L}-T_{S}\right)\right)^{2}\right]}{\sqrt{\pi}\left(T_{L}-T_{S}\right)} H_{f}
$$

where $H_{f}$ is the heat of fusion, $T_{S L}=\left(T_{S}+T_{L}\right) / 2$ is the average temperature of hardening (melting).

At transition from the liquid to solid metal there if the viscosity jump from some final value value $\mu_{L}$ in the melt to an very big value in the solid material $\mu_{S}$. The viscosity at (2) using function (4) was determined as:

$$
\mu=\mu_{L} f_{L}+\mu_{S}\left(1-f_{L}\right)
$$

When a mathematical determination $\mu_{S}$ is ready, then it will ensure freezing in a solid phase $\left(\mu_{S}>>\mu_{L}\right)$. Density, thermal conductivity and capacity of the material jumps due to phase transformation can be described in the same way.

The surface forces are considered by bulk forces in the following way:

- bulk force $F_{\mathrm{sv}}$ applied in a thin transition layer, where the density gradient is significantly different from zero;

- the value of $F_{s v}$ is proportional to the gradient of the function of the interface placement and surface curvature;

So, the bulk force expression is:

$$
\overrightarrow{\mathrm{F}}_{\mathrm{sv}}=-\left(\sigma k(\phi) \nabla \phi-\frac{\partial \sigma}{\partial T} \nabla_{s} T-p_{\partial} \vec{n}\right) \delta(\phi)
$$

where $\phi$ is the indicator function, $k(\phi)$ is the curvature of the interface,

$$
k(\phi)=\operatorname{div} \vec{n}, \quad \vec{n}=\frac{\nabla \phi}{|\nabla \phi|}, \quad n_{z}=\frac{1}{|\nabla \phi|} \frac{\partial \phi}{\partial z}
$$

$\delta(\phi)$ is delta function of Dirac, $\sigma$ is the melt surface tension coefficient.

The first summand at equation (11) is capillary force along the normal to the surface, the second term is for Marangoni effects, where $\nabla_{s} T$ is the tangential to the curved surface component gradient of the temperature. The third summand addresses the processes, which occur when the metal is exposed to the electron beam in case of electron-beam welding, and determines the force of vapour pressure in the gas-vapour channel formed by the concentrated electron beam, which is determined as a sum of partial pressures of the alloy elements $P_{\partial}=\sum_{1}^{n} P_{i}$, where $P_{i}^{0}$ is the partial pressure of the saturated vapour of the $i$-th element above the pure element, which can be expressed as the Clapeyron-Clausius equation. The activity of elements in the alloy is determined by the following formula: $a_{i}=X_{i} \cdot \gamma_{i}$, where $X_{i}$ is the atomic fraction of the $i$-th element, $\gamma_{i}$ is the activity coefficient of the $i$-th element in the alloy. In case of plasma arc, it can be determined according to equation [34]:

$$
p_{\partial}=2 k_{I} I_{a} \exp \left[-2\left(\left(x-V_{H} t\right)^{2}+y^{2}\right) / r^{2}\right]
$$

where $I$ is the arc current, $k_{I}$ is the electrodynamic parameter.

When using the Level-Set method, the interface is set to the zero value of the level function $\phi$. As for the function of the interface placement, the following form of a transfer equation is solved:

$$
\frac{\partial \phi}{\partial t}+\left(F_{p}+\vec{n} \cdot \vec{u}\right) \cdot|\nabla \phi|=0
$$

The values of density, viscosity or concentration are calculated according to the function of the interface placement. In particular, the expression for density is written as:

$\rho(\phi)=\rho_{1}+\left(\rho_{2}-\rho_{1}\right) H(\phi)$

where $\rho_{1}$ и $\rho_{2}$ are the average values of densities, $H(\phi)$ is the Heaviside function:

$H(\phi)=\left\{\begin{array}{lll}0 & \text { if }<0, & \text { air } \\ \frac{1}{2} & \text { if } \phi=0 \\ 1 & \text { if } \phi>0, & \text { metal }\end{array}\right.$

Numerical representation of the expression (16) is given as:

$H_{\varepsilon}(\phi)=\left\{\begin{array}{cc}0 & \text { if } \phi<-\varepsilon \\ \frac{1}{2}\left[1+\frac{\phi}{\pi}-\frac{1}{\pi} \sin (\pi \phi / \varepsilon)\right] & \text { if }|\phi| \leq \varepsilon \\ 1 & \text { if } \phi>\varepsilon\end{array}\right.$

The delta function from (6) and (11) is:

$\delta(\phi)=H_{\varepsilon}^{\prime}(\phi)=\frac{\partial}{\partial \phi} H_{\varepsilon}(\phi)$ 


$$
\delta(\phi)=\left\{\begin{array}{l}
\frac{1}{2 \varepsilon}\left[1+\cos \left(\frac{\pi \phi}{\varepsilon}\right)\right] \text { if }|\phi| \leq \varepsilon \\
0 \quad \text { otherwise }
\end{array}\right.
$$

The thickness of the transition layer is usually assumed to be $2 \varepsilon$ , where $\varepsilon=\alpha \Delta x$ and $\Delta x$ is the mesh size of the neighborhood's mesh, $\alpha$ is an integer

Outside the melting zone the convective velocity of media is supposed to be equal to 0 at all boundaries, thus, the overall velocity at all the boundaries is equal to $\vec{u}_{0}$. The velocity in the part where the wire enters the computational domain (Fig.2b) is equal to $\vec{u}_{1}$. For the function of the interface placement, the orthogonal derivative is supposed to be equal to 0 at al boundaries.

\section{Results and Discussions}

The first numerical implementation was carried out in a twodimensional description of process of the wire melting using concentrated and arc energy sources. COMSOL Multiphysics 4.5 application software package was utilized. The computational geometry is in Fig. 3.

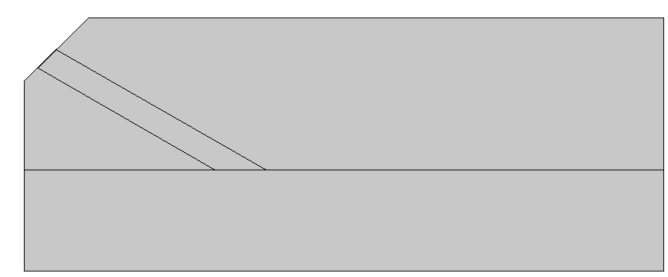

Fig. 3: The geometry of the computational domain

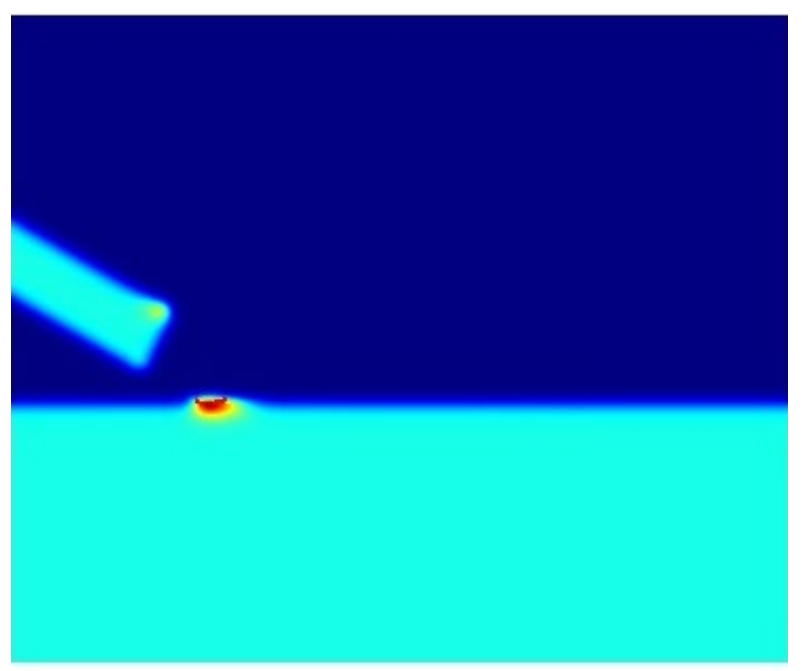

a)
The dimensions of the plate were $48 \mathrm{~mm}$ along $\mathrm{X}$ and $9 \mathrm{~mm}$ along Y. The two-dimensional grid was used. The grid had an uneven spacing (Fig.4): the maximal size of a node was $0.1 \mathrm{~mm}$ in the zone of heat source interaction and filler wire supply; over the remaining parts it was $0.5 \mathrm{~mm}$.

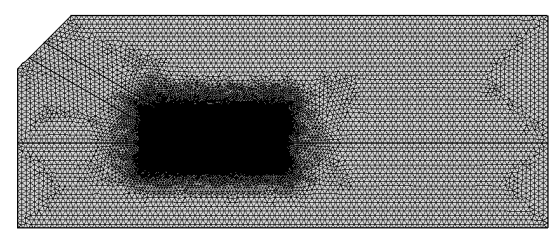

Fig.4: Grid-partitioning of the computational domain

Thermal and physical characteristics of the material, presented in Tab. 1 and variables of the regime presented in Tab. 2 were used as the initial parameters for the calculations.

Table 1: Thermal and physical properties of the deposited material

\begin{tabular}{|l|c|c|c|}
\hline \multicolumn{1}{|c|}{ Characteristics } & Notation & Unit of measurement & Value \\
\hline Liquidus temperature & $T_{L}$ & {$\left[{ }^{\circ} \mathrm{C}\right]$} & 1450 \\
\hline Solidus temperature & $T_{S}$ & {$\left[{ }^{\circ} \mathrm{C}\right]$} & 1520 \\
\hline Specific heat capacity & $C$ & {$[\mathrm{~J} \cdot \mathrm{kg}-1 \cdot \mathrm{K}-1]$} & 500 \\
\hline Density & $\rho$ & {$[\mathrm{kg} \cdot \mathrm{m}-3]$} & 7680 \\
\hline Thermal conductivity & $\lambda$ & {$\left[\mathrm{W} \cdot \mathrm{m}^{-1} \cdot \mathrm{K}^{-1}\right]$} & 28,9 \\
\hline Specific heat of melting & $H_{f}$ & $\mathrm{~kJ} / \mathrm{kg}$ & 84 \\
\hline
\end{tabular}

Table 2: Deposition mode

\begin{tabular}{|c|c|c|}
\hline Source power, $\mathrm{W}$ & Velocity, $\mathrm{mm} / \mathrm{s}$ & Feeding rate, $\mathrm{mm} / \mathrm{s}$ \\
\hline 810 & 5 & 16 \\
\hline
\end{tabular}

Fig. 5a presents the distribution of temperatures in the deposition zone in the beginning of the process $(t=0,01 \mathrm{c})$. The source of energy starts to melt the substrate and fed wire. At $t=0,1 \mathrm{~s}$ the drops of liquid metal start to move into the metal weld pool. At $\mathrm{t}=0.3 \mathrm{~s}$ the formation of the liquid metal pool has a spray metal transfer character. In $0.6 \mathrm{~s}$ after the beginning of the process, the liquid metal pool reaches its maximum size.

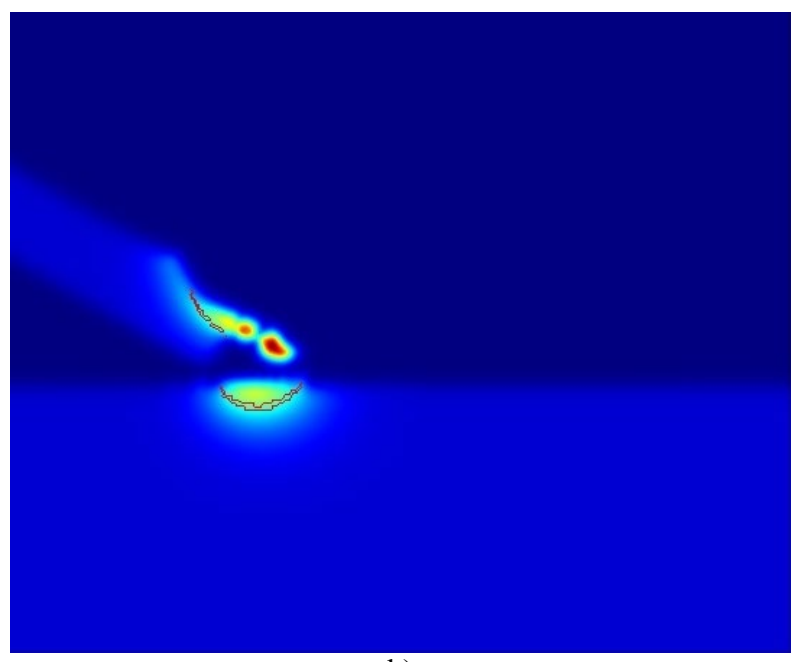

b) 


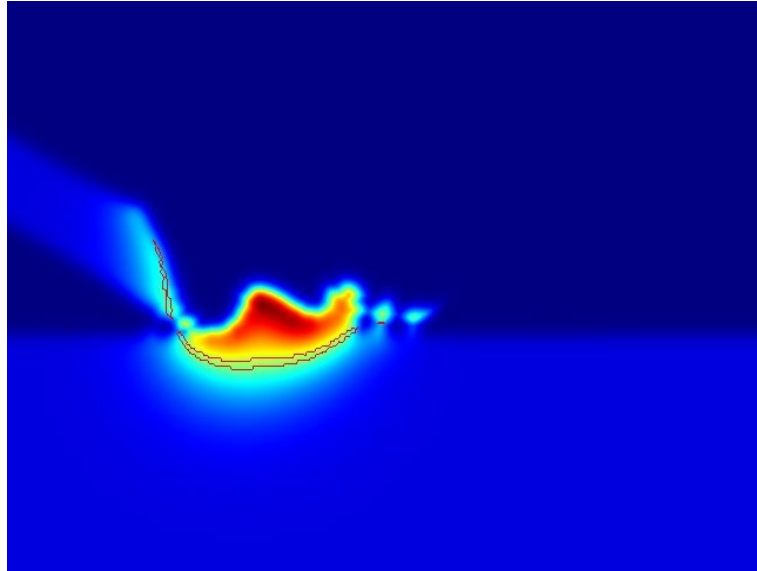

c)

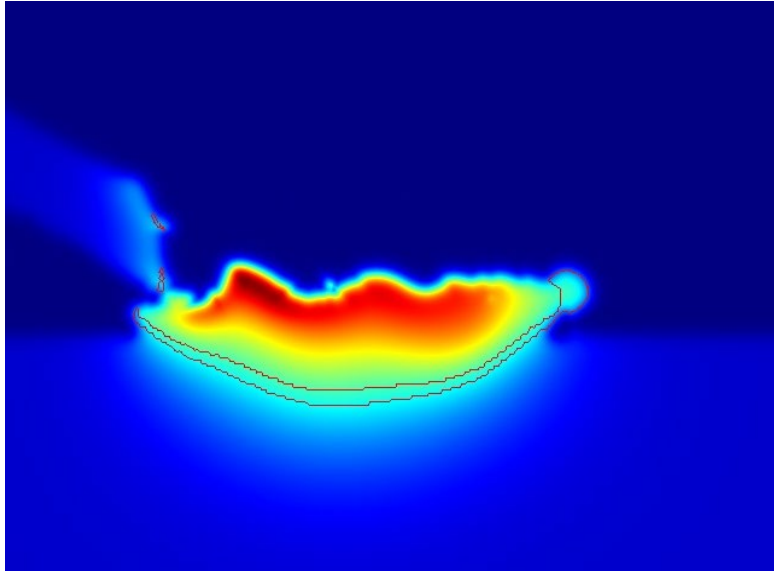

d)

Fig.5: The results of the preliminary numerical implementation of the heat and mass transfer model in a two-dimensional setting are as follows: a) $\mathrm{t}=0.01 \mathrm{~s}$; b) $\mathrm{t}=0.1 \mathrm{~s} ; \mathrm{c}$ ) $\mathrm{t}=0.3 \mathrm{~s} ; \mathrm{d}) \mathrm{t}=0.6 \mathrm{~s}$.

\section{Conclusion}

We have developed the heat and mass transfer mathematical model of during product wire-based additive manufacturing using concentrated power sources. The model includes equations for non- equilibrium conjugated processes of heat and mass transfer in free- surface liquidmetal, including the differential equations of fluid motion, term to the Marangoni effect on the melt surface, and calculates bulk distributions of temperatures, shape and size of the roll to be deposited. A preliminary numerical implementation has been carried out in a two-dimensional setting. The transition to the 3D-setting can be carried out by complicating the finite element model and transition to $3 \mathrm{D}$ elements.

\section{Acknowledgements}

The work was supported under the BRICS project by the Ministry of Education and Science of the Russian Federation (RFMEFI58317X0062) and MOST (No. 2017YFE0100100).

\section{References}

[1] D.H. Ding, Z.X. Pan, D. Cuiuri, et al., Wire-feed additive manufacturing of metal components: technologies, developments and future interests, International Journal of Advanced Manufacturing Technology, 81(1-4), (2015), 465-481.

[2] J. Ding, P. Colegrove, J. Mehnen, et al., Thermo-mechanical analysis of wire and arc additive layer manufacturing process on large multi-layer parts, Computational Materials Science, 50(12), (2011), 3315-3322.

[3] S.W. Williams, F. Martina, A.C. Addison, et al., Wire plus arc additive manufacturing, Materials Science and Technology, 32(7), (2016), 641-647.

[4] J. Xiong, Y.Y. Lei, H. Chen, et al., Fabrication of inclined thinwalled parts in multi-layer single-pass GMAW-based additive manufacturing with flat position deposition, Journal of Materials Processing Technology, 240, (2017), 397-403.

[5] M. P. Mughal, H. Fawad, R.A. Mufti, et al., Deformation modelling in layered manufacturing of metallic parts using gas metal arc welding: effect of process parameters, Modelling And Simulation In Materials Science And Engineering, 13(7), (2005), 187-204.

[6] A. Vasinonta, J.L. Beuth, M. Griffith, Process maps for predicting residual stress and melt pool size in the laser-based fabrication of thin-walled structures, Journal of Manufacturing Science and Engineering, 129(1), (2007), 101-109.

[7] V. Manvatkar, A. De, T. Deb Roy, Heat transfer and material flow during laser assisted multi-layer additive manufacturing, Journal of Applied Physics, 116(12), (2014).

[8] L.E. Svensson, B. Gretoft, H. Bhadeshia, An analysis of cooling curves from the fusion zone of steel weld deposits, Scandinavian Journal of Metallurgy, 15(2), (1986), 97-103.
[9] I. M. Mark, C. Korner, Multiscale modeling of powder bed-based additive manufacturing, Annual Review of Materials Research, 46, (2016), 93-123.

[10] T. Amine, J.W. Newkirk, F. Liou. Investigation of effect of process parameters on multilayer builds by direct metal deposition, Applied Thermal Engineering, 73(1), (2014), 500-511.

[11] Y.P. Hu, C.W. Chen, K. Mukherjee, Measurement of temperature distributions during laser cladding process, Journal of Laser Applications, 12(3), (2000), 126-130.

[12] I.V. Shishkovsky, V.I. Scherbakov, Y.G. Morozov, et al., Surface laser sintering of exothermic powder compositions, Journal of Thermal Analysis and Calorimetry, 91(2), (2008), 427-436.

[13] J. Benda, Temperature controlled selective laser sintering. Solid Freeform Fabrication Symposium, Austin, 1994, 277-284.

[14] M. Doubenskaia, M. Pavlov, Y. Chivel, Optical system for on-line monitoring and temperature control in selective laser melting technology, Key engineering materials, 437, (2010), 458-461.

[15] D. Hu., R. Kovacevic, Sensing, modeling and control for laserbased additive manufacturing, International Journal of Machine Tools and Manufacture, 43(1), (2003), 51-60.

[16] X. Jian, S. Jinghua, G. Yiqingc, Novel measurement method for selective laser sintering transient temperature field, 3rd International symposium on advanced optical manufacturing and testing technologies: optical test and measurement technology and equipment, (2007), 67234.

[17] S. Price, K. Cooper, K. Chou, Evaluations of temperature measurements by near-infrared thermography in powder-based electron-beam additive manufacturing, International Journal of Rapid Manufacturing (IJRAPIDM), 4(1), (2014), 761-773

[18] J.U. Brackbill, D.B.Kothe, C. Zemach, A continuum method for modeling surface tension, Journal of Computational Physics, 100 , (1992), 335-354.

[19] M. Sussman, P. Smereka, S. Osher, A level set approach for computing solutions to incompressible two-phase flow, Journal of computational physics, 114, (1994), 146-159.

[20] M. Sussman, E. Fatemi, P. Smereka, et al., An improved level set method for incompressible two-phase flow, Computers \& Fluids, 27(5-6), (1998), 663-680.

[21] J. Mazumder, H. Qi, H. Ki, Numerical simulation of heat transfer and fluid flow in coaxial laser cladding process for direct metal deposition, Journal of Applied Physics, 100(2), (2006), 024903.

[22] F. Kong, H. Zhang, G. Wang, Modeling of heat transfer, fluid flow and solute diffusion in the plasma deposition manufacturing functionally gradient materials, PIERS Proceedings, Moscow, Russia, August 18-21, 2009,1948-1952.

[23] I. Tomashchuk, P. Sallamand, J.M. Jouvard,et al., The simulation of morphology of dissimilar copper-steel electron beam welds using level set method, Computational Materials Science, 48(4), (2010), 827-836.

[24] V. Manvatkar, A. De, T. Deb Roy, Spatial variation of melt pool geometry, peak temperature and solidification parameters during laser assisted additive manufacturing process, Materials Science and Technology, 31(8), (2015), 924-930.

[25] A. Raghavan, H.L. Wei, T.A. Palmer, et al., Heat transfer and fluid flow in additive manufacturing, Journal of Laser Applications, 25(5), (2013), 052006. 
[26] S. Patankar, Numerical heat transfer and fluid flow. CRC Press, New York, 1980.

[27] S.A. David, T. Deb Roy, Current issues and problems in welding science, Science, 257(5069), (1992), 497-502.

[28] T. Deb Roy, S.A. David, Physical processes in fusion welding, Reviews of Modern Physics, 67(1), (1995), 85-112.

[29] I. A. Kharitonov, V.N. Martynov, V. K. Shcherbakov, et al., The Second International Conference on Electron Beam Welding and Related Technologies, National Research University "Moscow Power Engineering Institute", November 14-17, 2017, Book of materials and reports, MPEI Publishing House, Moscow, 2017, 257-265.

[30] X. He, J. Mazumder, Transport phenomena during direct metal deposition, Journal of Applied Physics 101(5) (2007) 053113.

[31] S. Morville, M. Carin, P. Peyre, et al., 2D longitudinal modeling of heat transfer and fluid flow during multilayered direct laser metal deposition process, Journal of Laser Applications, 24(3), (2012).

[32] S.Y. Wen, Y.C. Shin, Modeling of transport phenomena during the coaxial laser direct deposition process, Journal of Applied Physics, 108(4), (2010).

[33] A.I. Tsaplin, I.L. Nikulin, Modelling of thermophysical processes and objects in metal industry: coursebook, Publishing house of Perm State Technical University, Perm, 2011.

[34] E. Strakhova, V.A. Erofeev, V.A. Sudnik, Physical and Mathematical Modeling of Wide-Layer Cladding with Transverse Oscillations of Plasma, Welding and Diagnostics, 3, (2009), 32-38. 